\title{
Supplemental Information for SIBaR: A New Method for Background Quantification and Removal from Mobile Air Pollution Measurements
}

Blake Actkinson ${ }^{1}$, Katherine Ensor ${ }^{2}$, Robert J. Griffin ${ }^{1,3}$

${ }^{1}$ Department of Civil and Environmental Engineering, Rice University, Houston, TX 77005, USA

${ }^{2}$ Department of Statistics, Rice University, Houston, TX 77005, USA

${ }^{3}$ Department of Chemical and Biomolecular Engineering, Rice University, Houston, TX 77005, USA

\begin{tabular}{|c|c|c|c|c|}
\hline Census Tracts & $\begin{array}{l}\text { Population } \\
\text { Total }\end{array}$ & $\begin{array}{l}\text { \# Metal } \\
\text { Recyclers }\end{array}$ & $\begin{array}{l}\text { \# Concrete Batch } \\
\text { Plants }\end{array}$ & $\begin{array}{l}\text { \# Petrochemical } \\
\text { Facilities }\end{array}$ \\
\hline Northwest Domain & 34873 & 7 & 2 & $\mathbf{0}$ \\
\hline North Spring Branch & 5126 & 0 & 0 & 0 \\
\hline South Spring Branch & 3604 & 0 & 0 & 0 \\
\hline Memorial Park & 6908 & 0 & 0 & 0 \\
\hline Washington Corridor & 5432 & 2 & 0 & 0 \\
\hline North River Oaks & 1803 & 0 & 0 & 0 \\
\hline South River Oaks & 2775 & 0 & 0 & 0 \\
\hline West Eastex & 2753 & 5 & 2 & 0 \\
\hline North Heights & 6472 & 1 & 0 & 0 \\
\hline Southwest Domain & 24927 & $\mathbf{0}$ & 1 & $\mathbf{0}$ \\
\hline Westchase & 5548 & 0 & 0 & 0 \\
\hline Sharpstown & 5616 & 0 & 0 & 0 \\
\hline Sharpstown North & 3484 & 0 & 1 & 0 \\
\hline Sharpstown South & 5196 & 0 & 0 & 0 \\
\hline Bayland Park & 5083 & 0 & 0 & 0 \\
\hline $\begin{array}{l}\text { South Beltway } \\
\text { Central Domain }\end{array}$ & 2530 & 3 & 8 & $\mathbf{0}$ \\
\hline $\begin{array}{r}\text { South Beltway } \\
\text { Central }\end{array}$ & 2530 & 3 & 8 & 0 \\
\hline Rice Domain & 8247 & $\mathbf{0}$ & $\mathbf{0}$ & $\mathbf{0}$ \\
\hline North Rice & 2892 & 0 & 0 & 0 \\
\hline South Rice & 5355 & 0 & 0 & 0 \\
\hline $\begin{array}{l}\text { Ship Channel } \\
\text { Domain }\end{array}$ & 20177 & 4 & 1 & 4 \\
\hline Clinton & 2127 & 2 & 1 & 1 \\
\hline West Galena Park & 5245 & 0 & 0 & 0 \\
\hline East Galena Park & 3000 & 0 & 0 & 0 \\
\hline Manchester & 1647 & 0 & 0 & 1 \\
\hline Harrisburg & 1496 & 2 & 0 & 2 \\
\hline Milby Park & 6662 & 0 & 0 & 0 \\
\hline
\end{tabular}

Table S1. Neighborhood summary table which includes the total population and number of important point source emitters within each neighborhood. Data courtesy of the U.S. Census and Environmental Defense Fund (Census 2010; Environmental Defense Fund). Domain names refer to direction from downtown or local landmarks (such as Rice University). 


\section{Parameter}

NO

$\mathrm{NO}_{2}$

$\mathrm{CO}_{2}$

$\mathrm{BC}$
Instrument

T200 NO Analyzer

T500U $\mathrm{NO}_{2}$ Analyzer

Li-COR $\mathrm{CO}_{2}$ Analyzer

AE33 Aethalometer

Table S2. Instruments used in campaign to make measurements.

\begin{tabular}{|c|c|c|c|c|c|c|c|c|c|c|c|c|}
\hline & 1 & 2 & 3 & 4 & 5 & 6 & 7 & 8 & 9 & 10 & 11 & 12 \\
\hline 1 & 0.0000 & 0.0017 & 0.0040 & 0.0113 & 0.0145 & 0.0146 & 0.0153 & 0.0059 & 0.0059 & 0.0164 & 0.0115 & 0.0068 \\
\hline 2 & & 0.0000 & 0.0041 & 0.0112 & 0.0143 & 0.0147 & 0.0150 & 0.0058 & 0.0058 & 0.0163 & 0.0114 & 0.0075 \\
\hline 3 & & & 0.0000 & 0.0101 & 0.0147 & 0.0146 & 0.0151 & 0.0042 & 0.0042 & 0.0154 & 0.0118 & 0.0081 \\
\hline 4 & & & & 0.0000 & 0.0154 & 0.0111 & 0.0158 & 0.0120 & 0.0119 & 0.0167 & 0.0130 & 0.0142 \\
\hline 5 & & & & & 0.0000 & 0.0087 & 0.0039 & 0.0151 & 0.0152 & 0.0194 & 0.0183 & 0.0168 \\
\hline 6 & & & & & & 0.0000 & 0.0087 & 0.0153 & 0.0153 & 0.0196 & 0.0181 & 0.0165 \\
\hline 7 & & & & & & & 0.0000 & 0.0148 & 0.0148 & 0.0192 & 0.0195 & 0.0168 \\
\hline 8 & & & & & & & & 0.0000 & 0.0003 & 0.0144 & 0.0128 & 0.0069 \\
\hline 9 & & & & & & & & & 0.0000 & 0.0143 & 0.0128 & 0.0068 \\
\hline 10 & & & & & & & & & & 0.0000 & 0.0168 & 0.0161 \\
\hline 11 & & & & & & & & & & & 0.0000 & 0.0135 \\
\hline 12 & & & & & & & & & & & & 0.0000 \\
\hline
\end{tabular}

Table S3. Pairwise RMSE values between different iterations of SIBaR. 

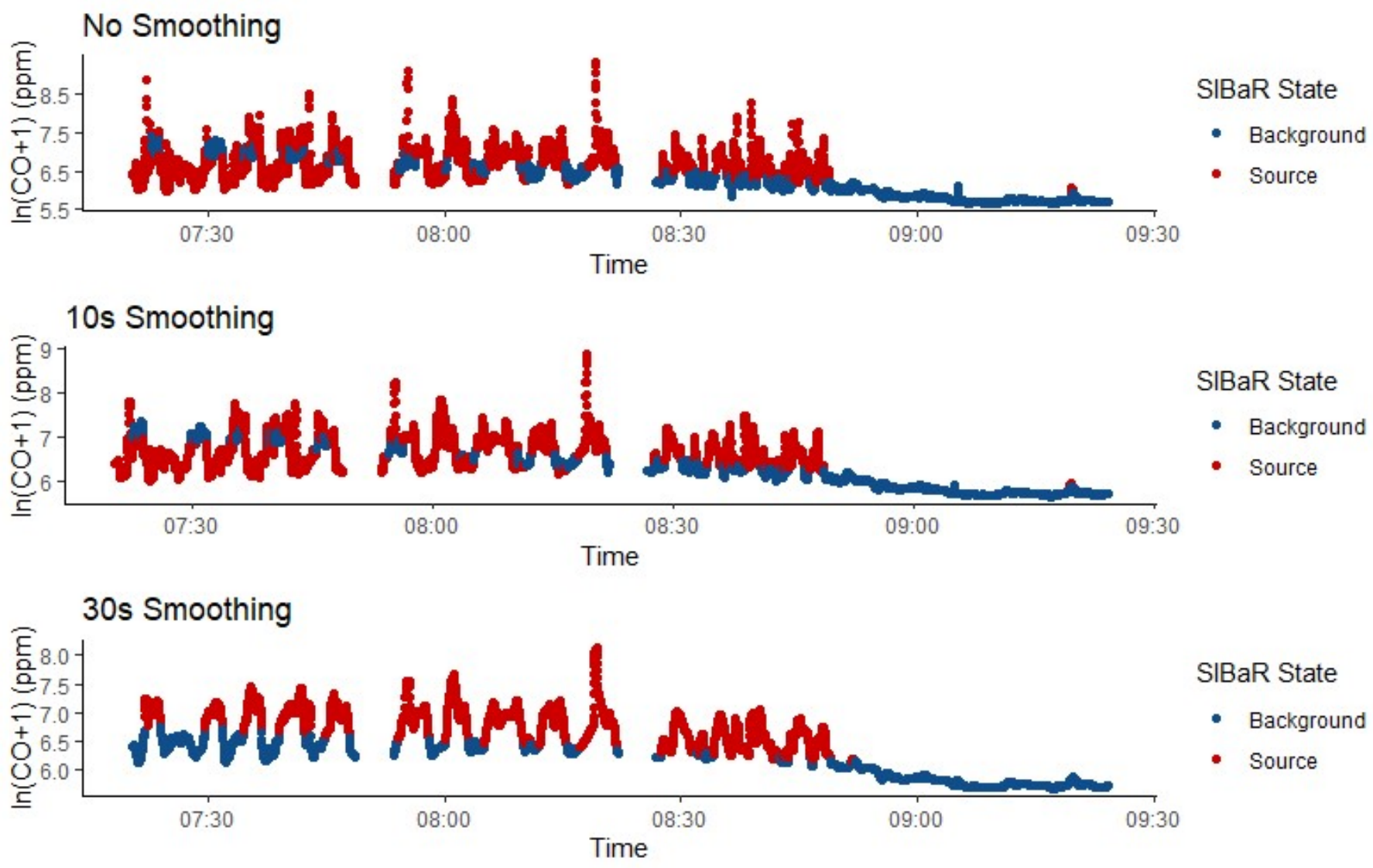

Figure S1. SIBaR state designation time window sensitivity. 

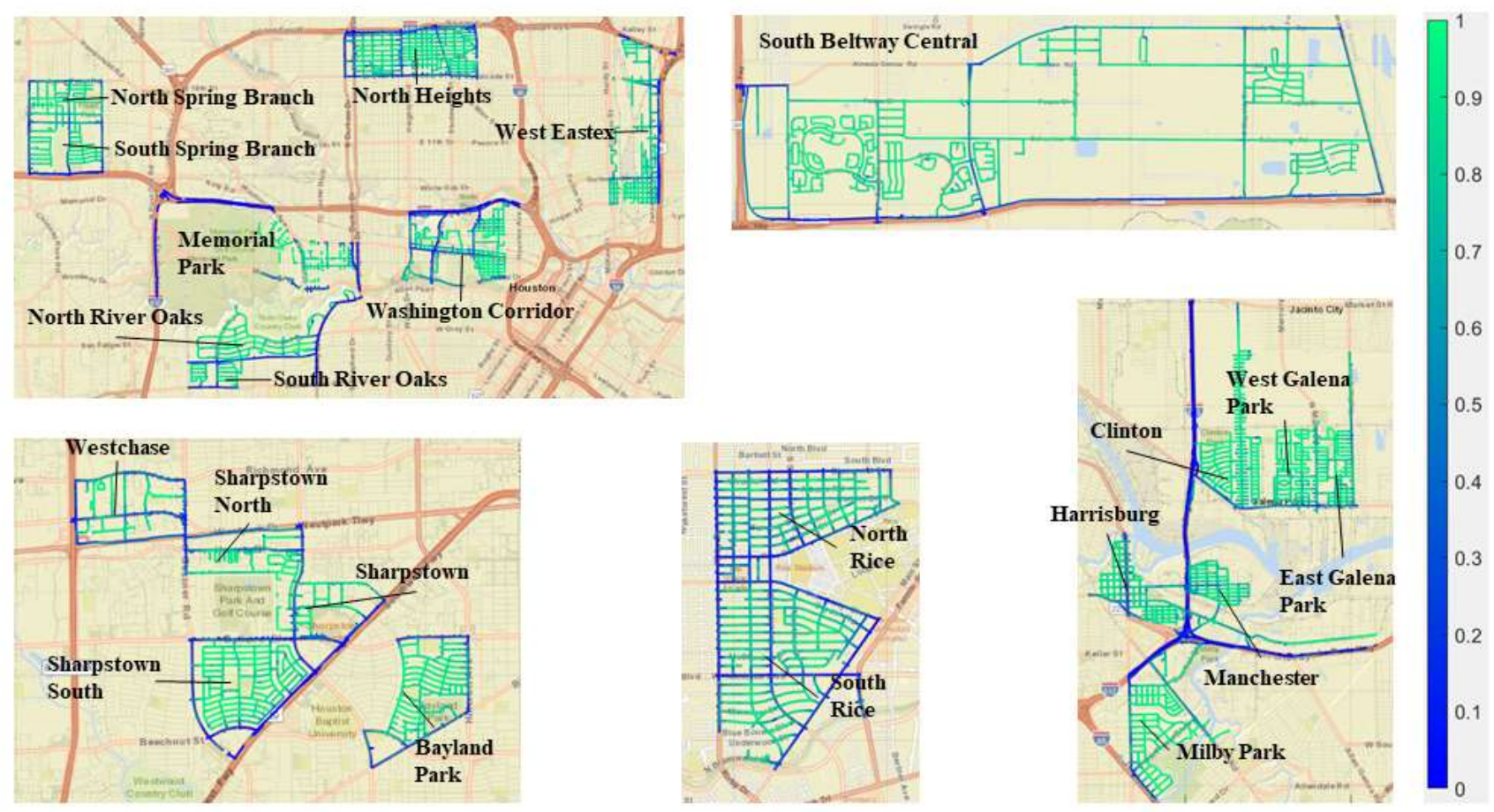

Figure $\mathrm{S}_{1} \mathrm{CO}_{2}$ mapped fractional contributions. A value of 1 implies all measurements reflect background, while a value of 0 implies all measurements reflect non-background. Basemap generated by Matlab geobasemap 'streets' and is hosted by ESRI (Sources: Esri, DeLorme, HERE, USGS, Intermap, iPC, NRCAN, Esri Japan, METI, Esri China (Hong Kong), Esri (Thailand), MapmyIndia, Tomtom).

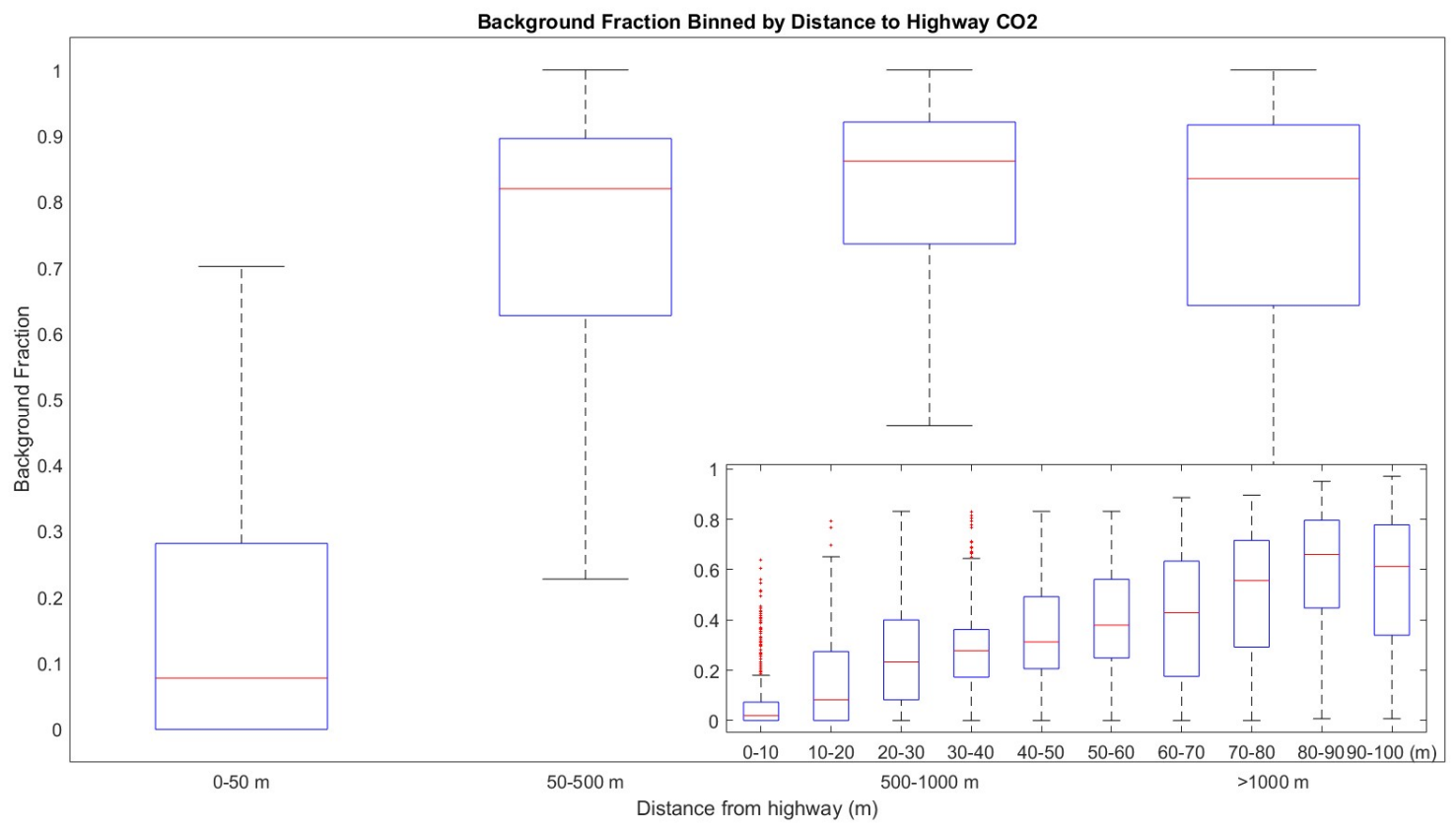

Figure $\mathrm{S3}_{2} \mathrm{CO}_{2}$ background fraction boxplot binned by distance. 

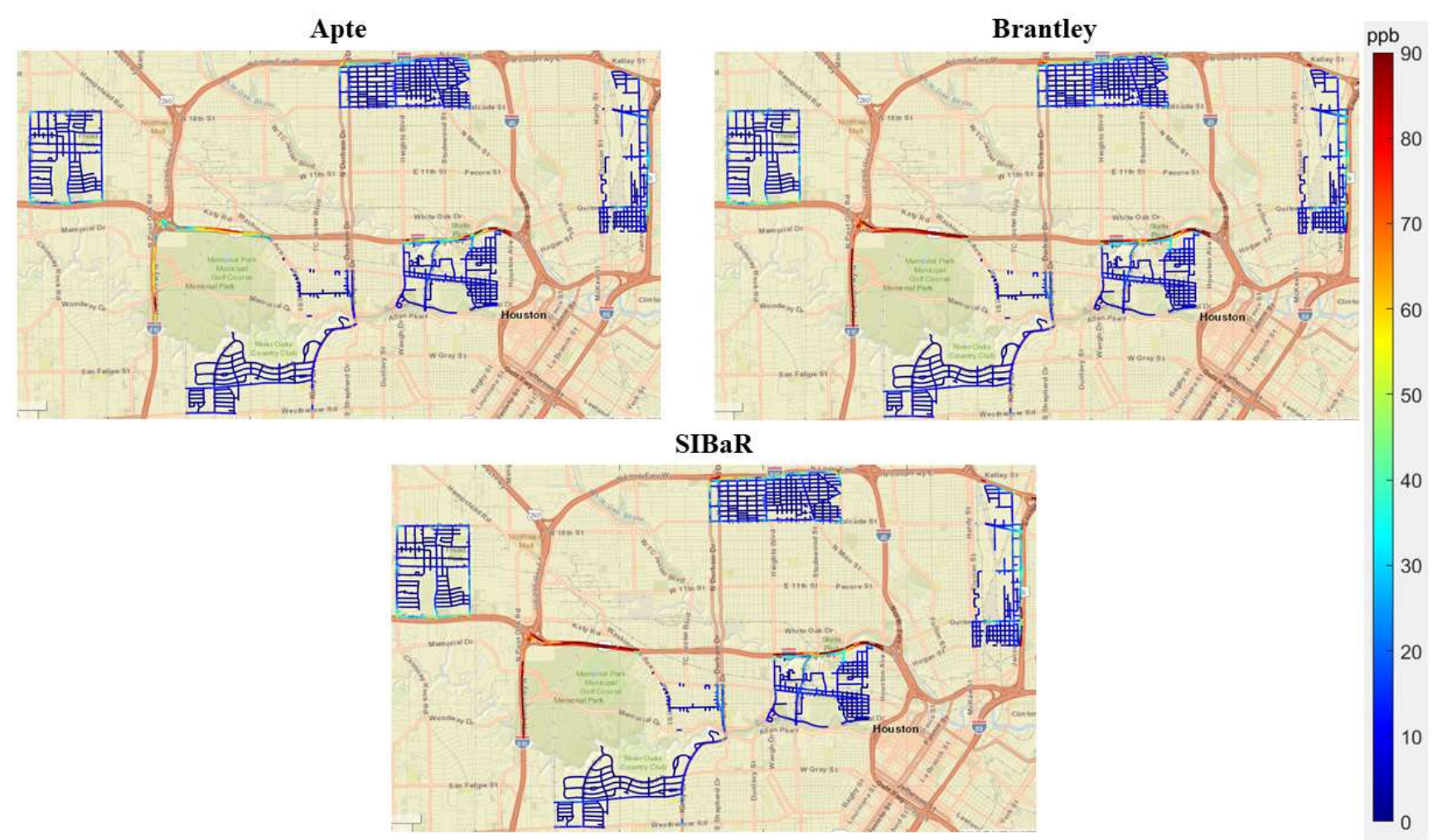

Figure S4. Mapped median NOx source contributions. Northwest quadrant. Basemap generated by Matlab geobasemap 'streets' and is hosted by ESRI (Sources: Esri, DeLorme, HERE, USGS, Intermap, iPC, NRCAN, Esri Japan, METI, Esri China (Hong Kong), Esri (Thailand), MapmyIndia, Tomtom).
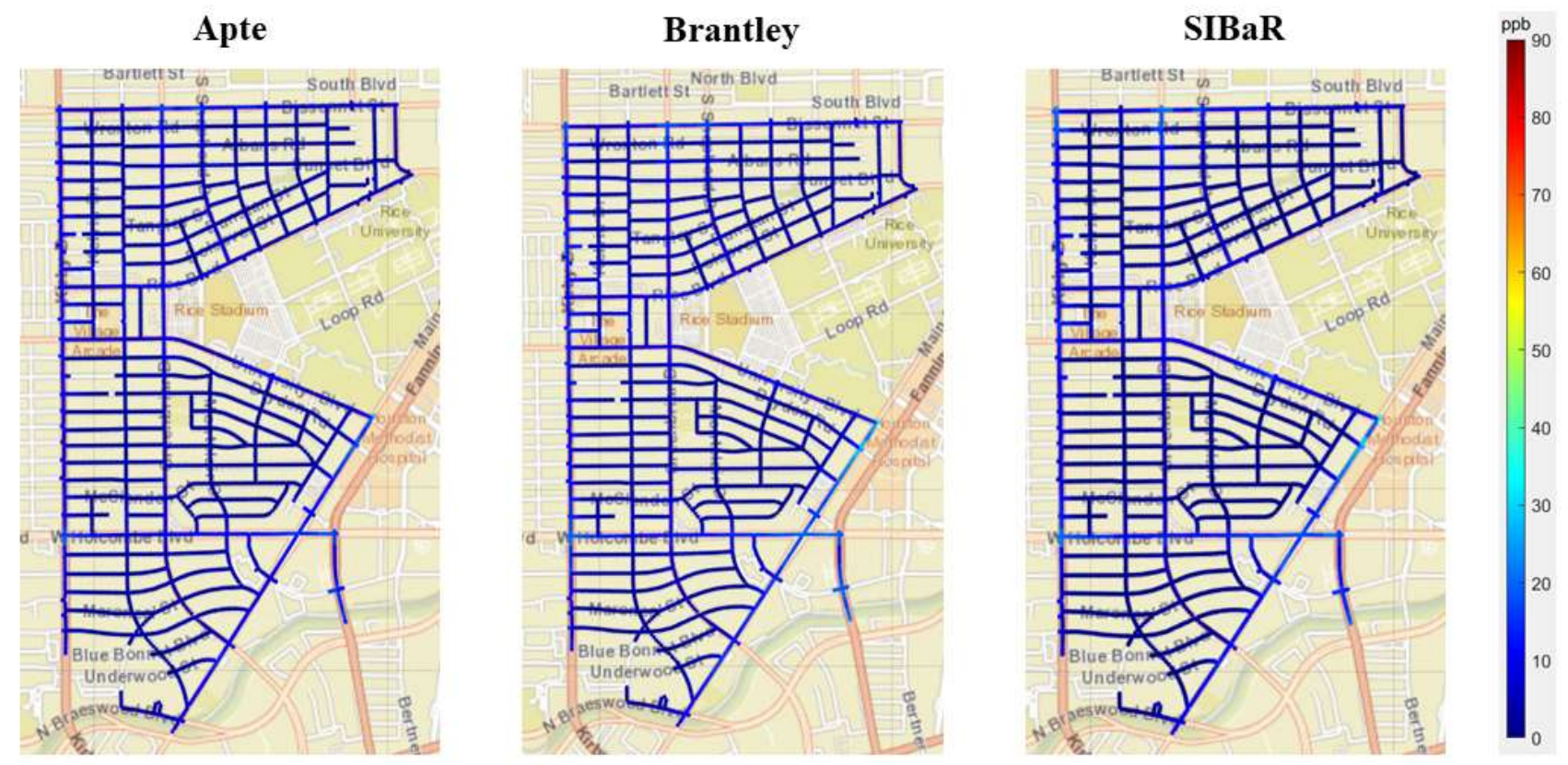

Figure S5. Mapped median NO ${ }_{x}$ source contributions. North and South Rice. Basemap generated by Matlab geobasemap 'streets' and is hosted by ESRI (Sources: Esri, DeLorme, HERE, USGS, Intermap, iPC, NRCAN, Esri Japan, METI, Esri China (Hong Kong), Esri (Thailand), MapmyIndia, Tomtom). 


\section{Apte}

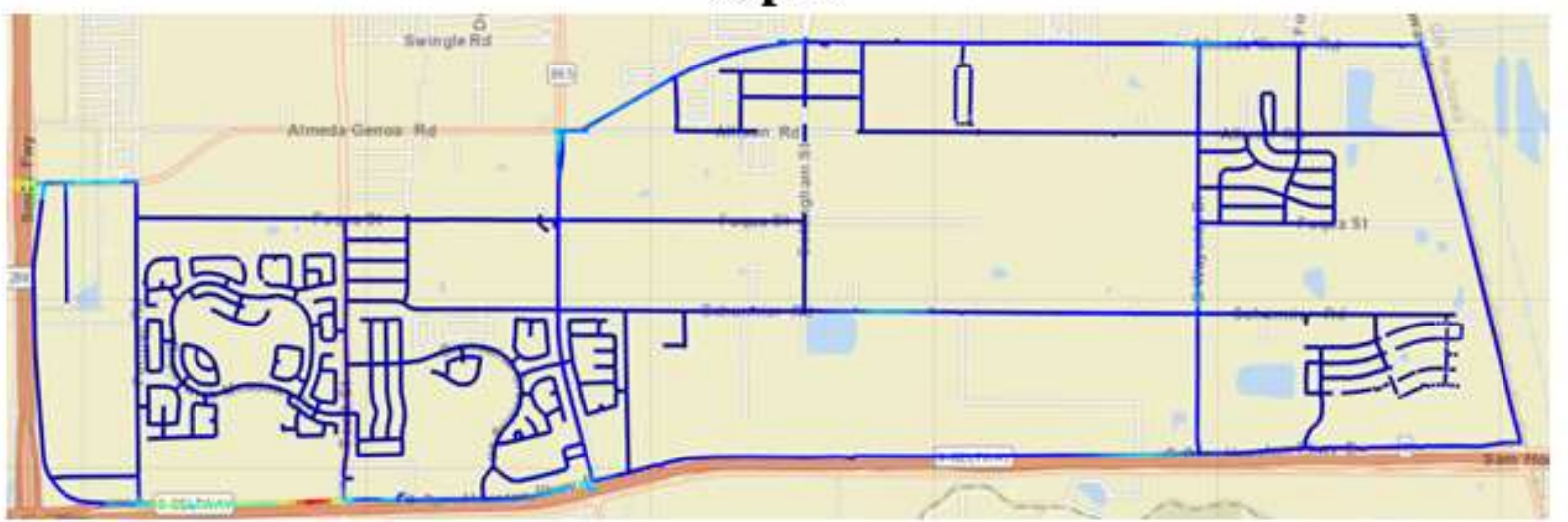

Brantley

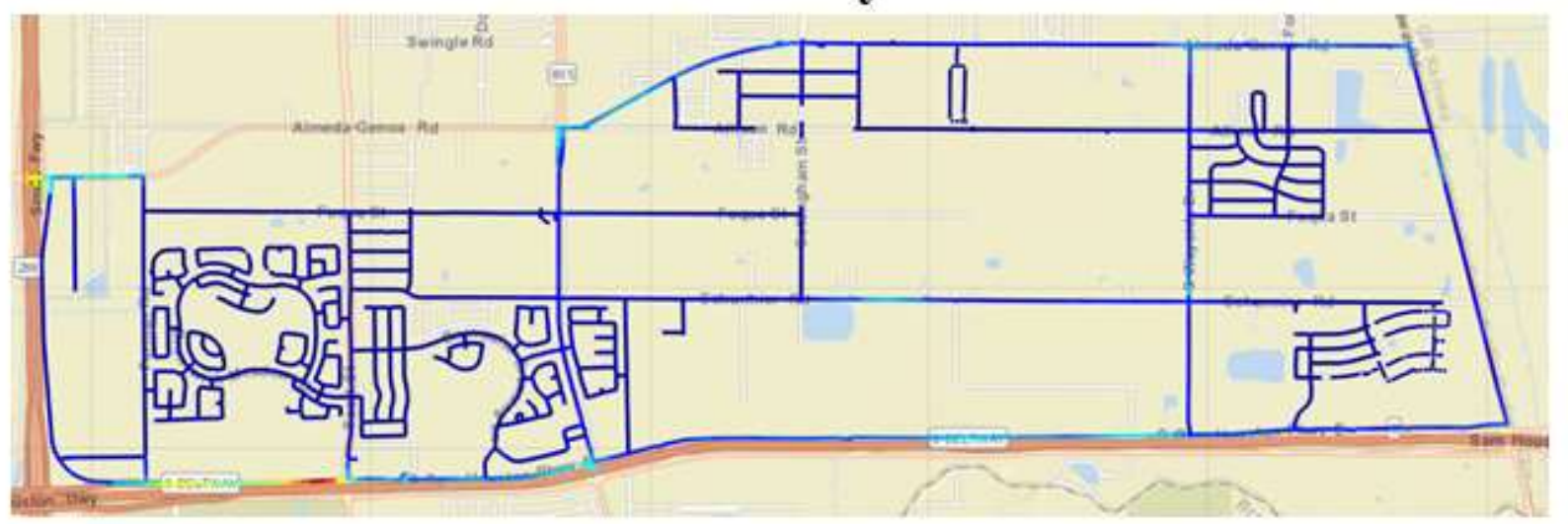

SIBaR

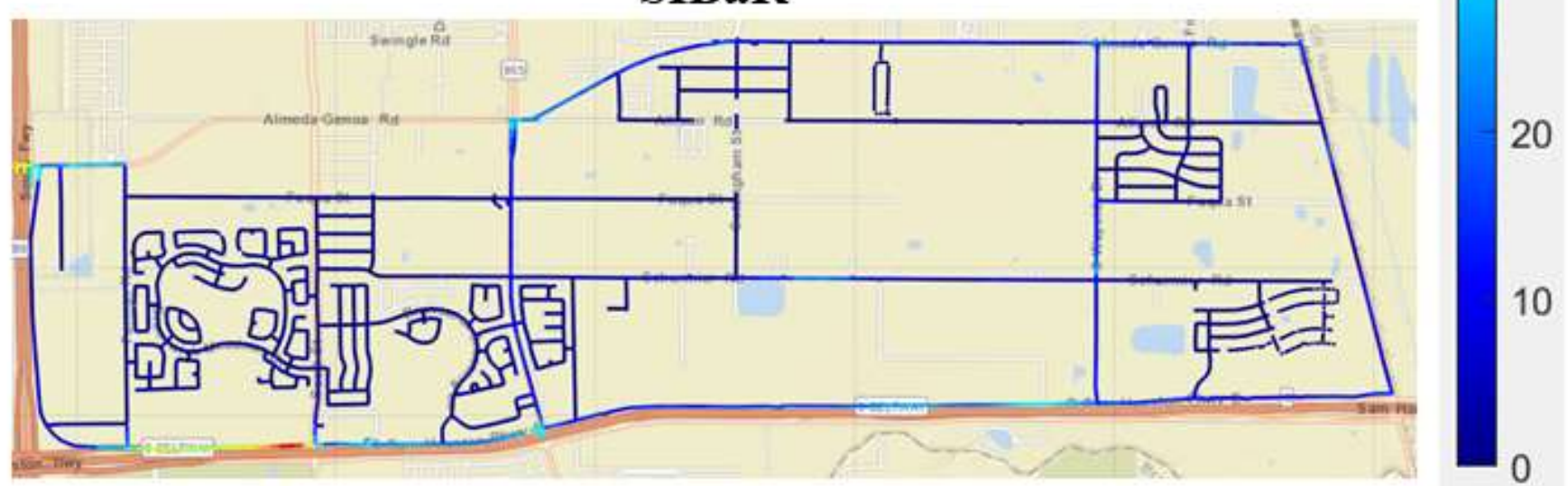

Figure S6. Mapped median NO $\mathrm{x}_{\mathrm{x}}$ source contributions. South Beltway Central. Basemap generated by Matlab geobasemap 'streets' and is hosted by ESRI (Sources: Esri, DeLorme, HERE, USGS, Intermap, iPC, NRCAN, Esri Japan, METI, Esri China (Hong Kong), Esri (Thailand), MapmyIndia, Tomtom). 


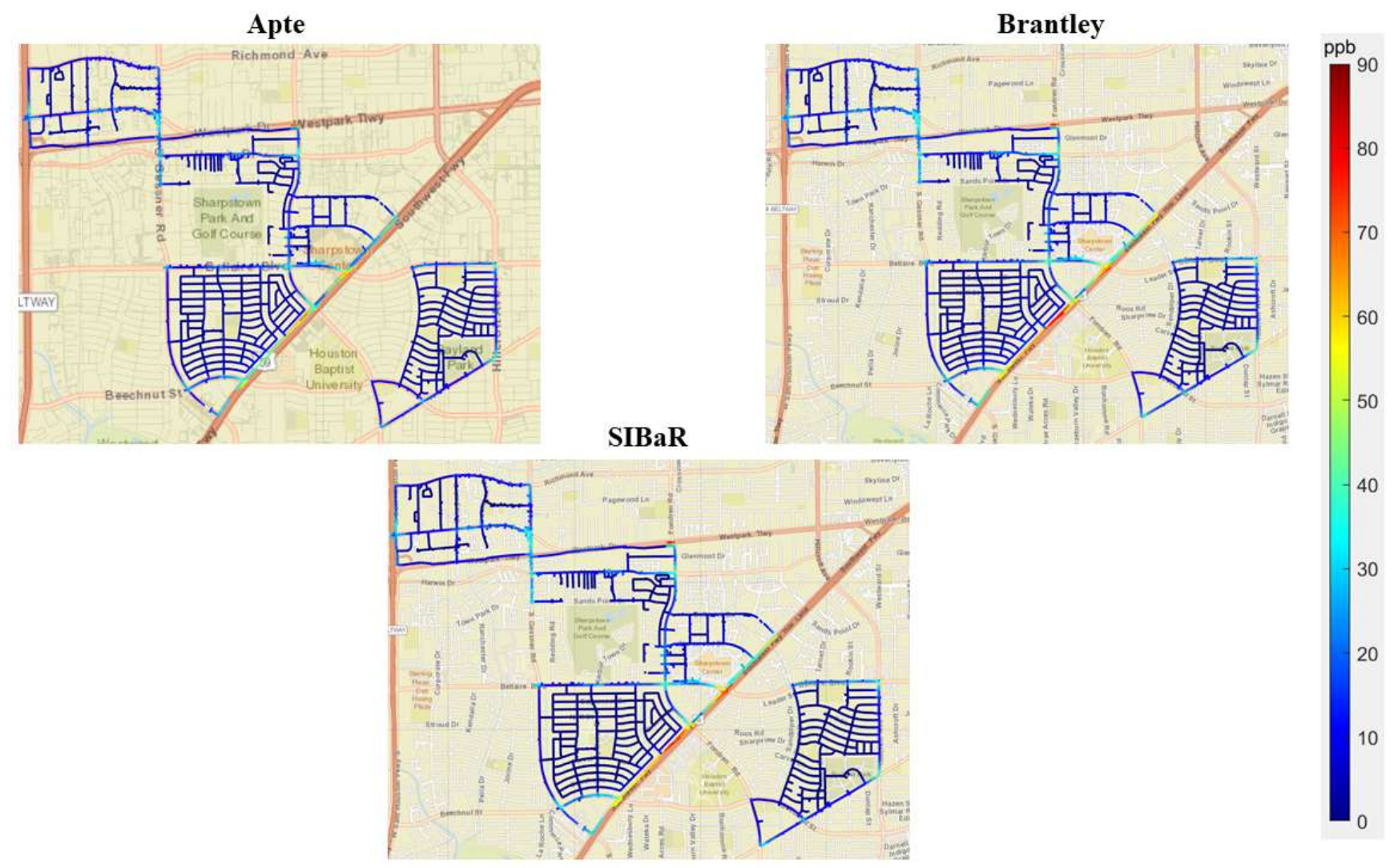

Figure S7. Mapped median NOx source contributions. Southwest quadrant. Basemap generated by Matlab geobasemap 'streets' and is hosted by ESRI (Sources: Esri, DeLorme, HERE, USGS, Intermap, iPC, NRCAN, Esri Japan, METI, Esri China (Hong Kong), Esri (Thailand), MapmyIndia, Tomtom).
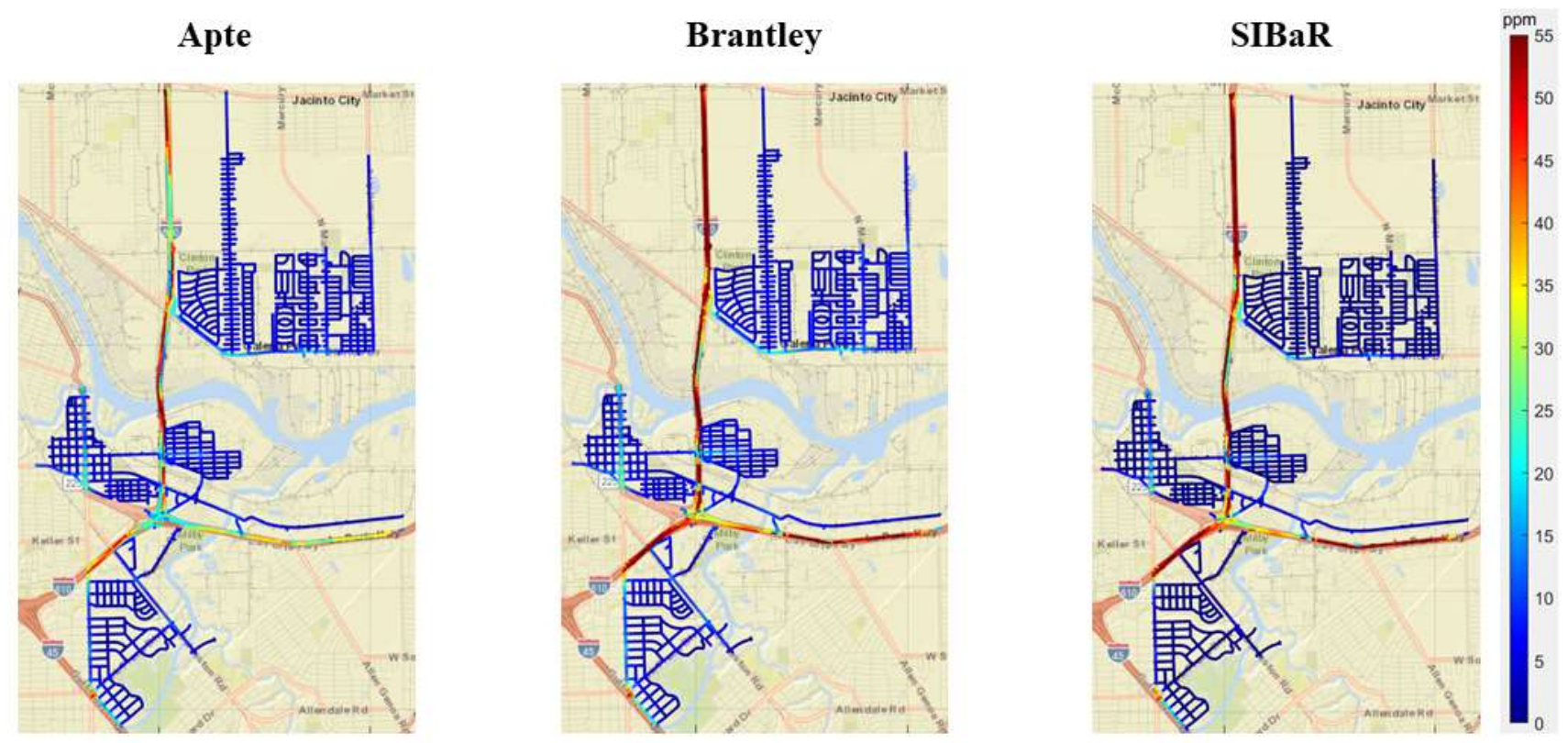

Figure S8. $\mathrm{CO}_{2}$ mapped median source contributions. Ship Channel. Basemap generated by Matlab geobasemap 'streets' and is hosted by ESRI (Sources: Esri, DeLorme, HERE, USGS, Intermap, iPC, NRCAN, Esri Japan, METI, Esri China (Hong Kong), Esri (Thailand), MapmyIndia, Tomtom). 


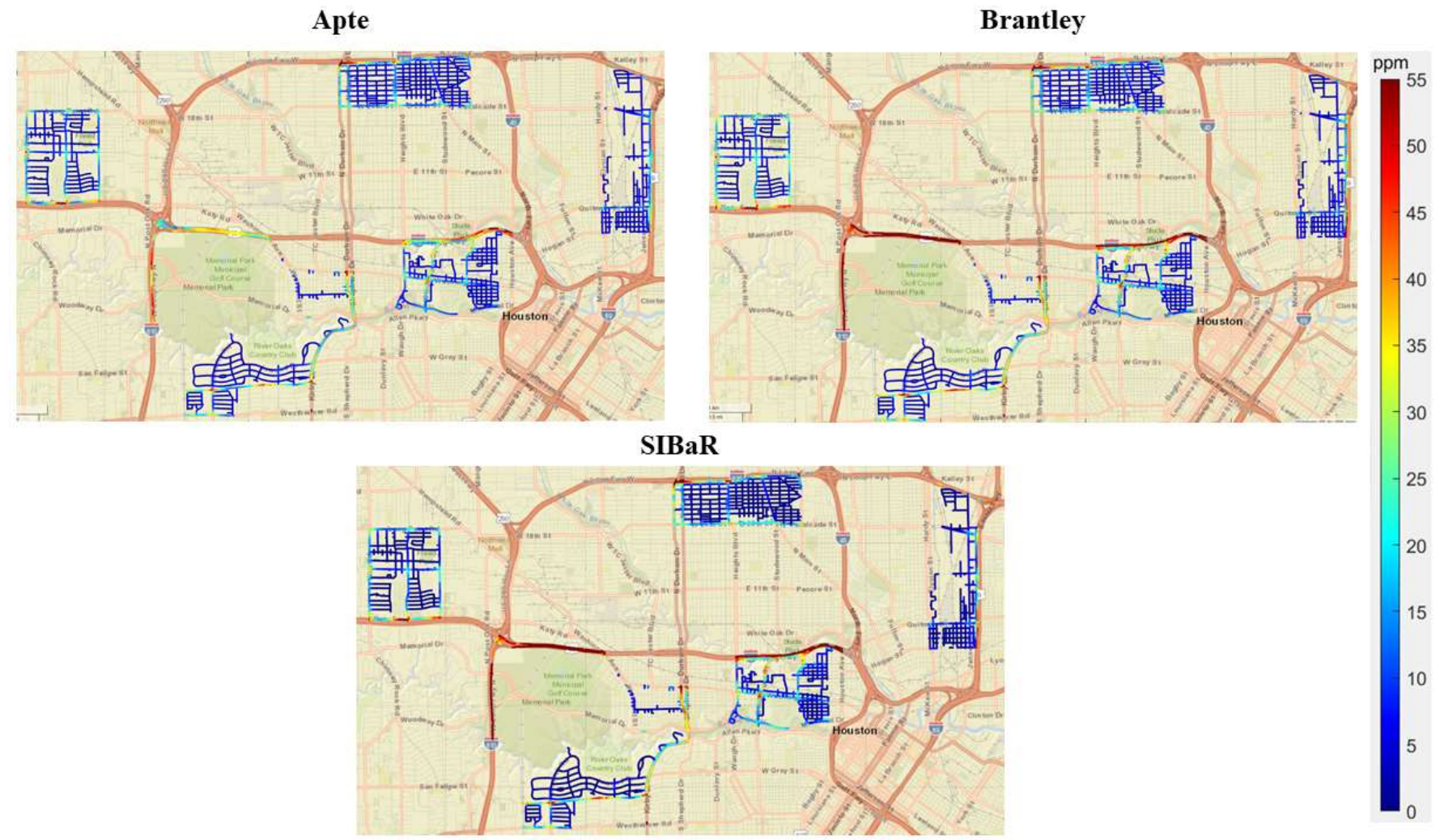

Figure $\mathrm{S9} . \mathrm{CO}_{2}$ mapped median source contributions. Northwest domain. Basemap generated by Matlab geobasemap 'streets' and is hosted by ESRI (Sources: Esri, DeLorme, HERE, USGS, Intermap, iPC, NRCAN, Esri Japan, METI, Esri China (Hong Kong), Esri (Thailand), MapmyIndia, Tomtom).
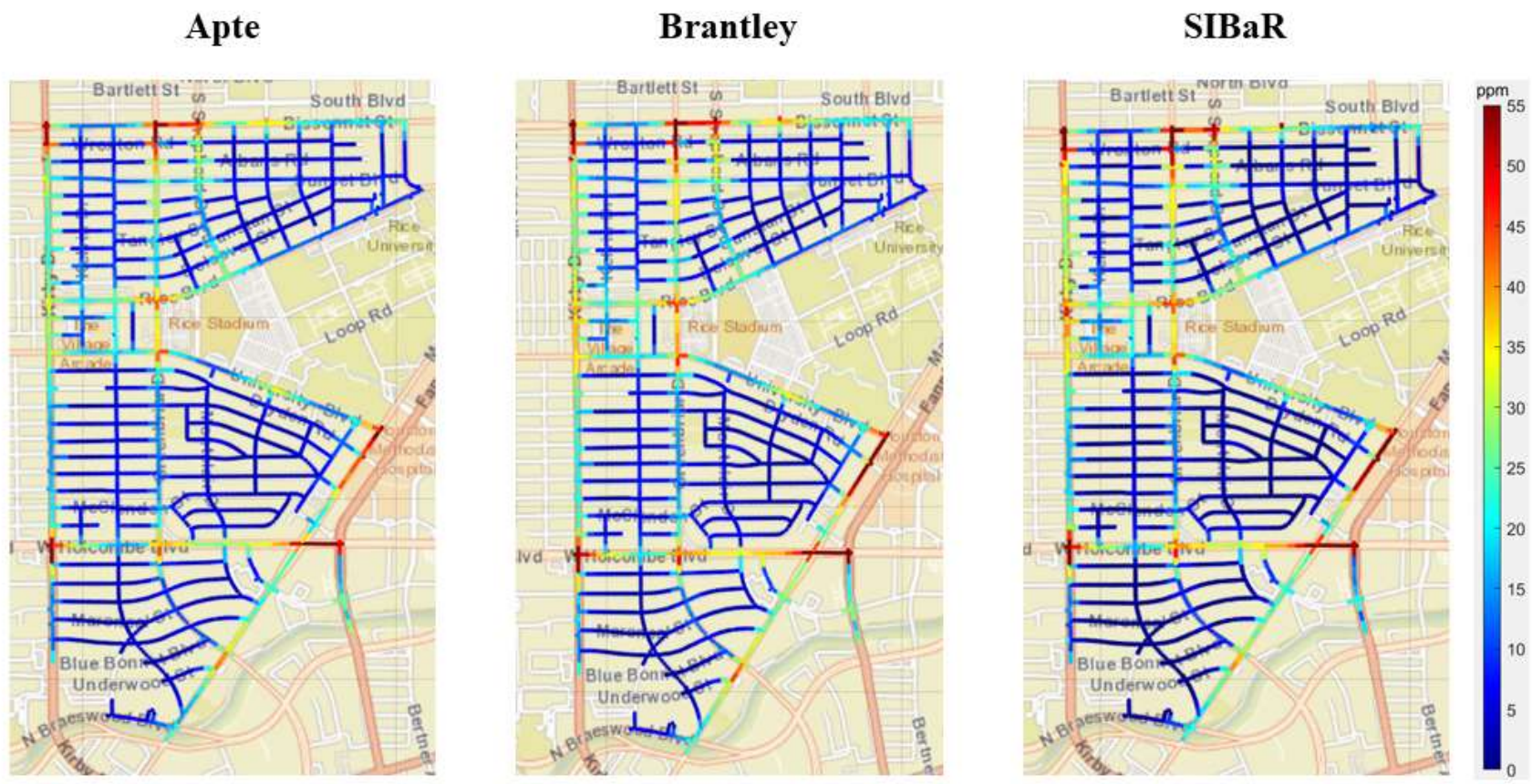

Figure $\mathrm{S} 10 .^{\mathrm{CO}_{2}}$ mapped median source contributions. North and South Rice. Basemap generated by Matlab geobasemap 'streets' and is hosted by ESRI (Sources: Esri, DeLorme, HERE, USGS, Intermap, iPC, NRCAN, Esri Japan, METI, Esri China (Hong Kong), Esri (Thailand), MapmyIndia, Tomtom). 


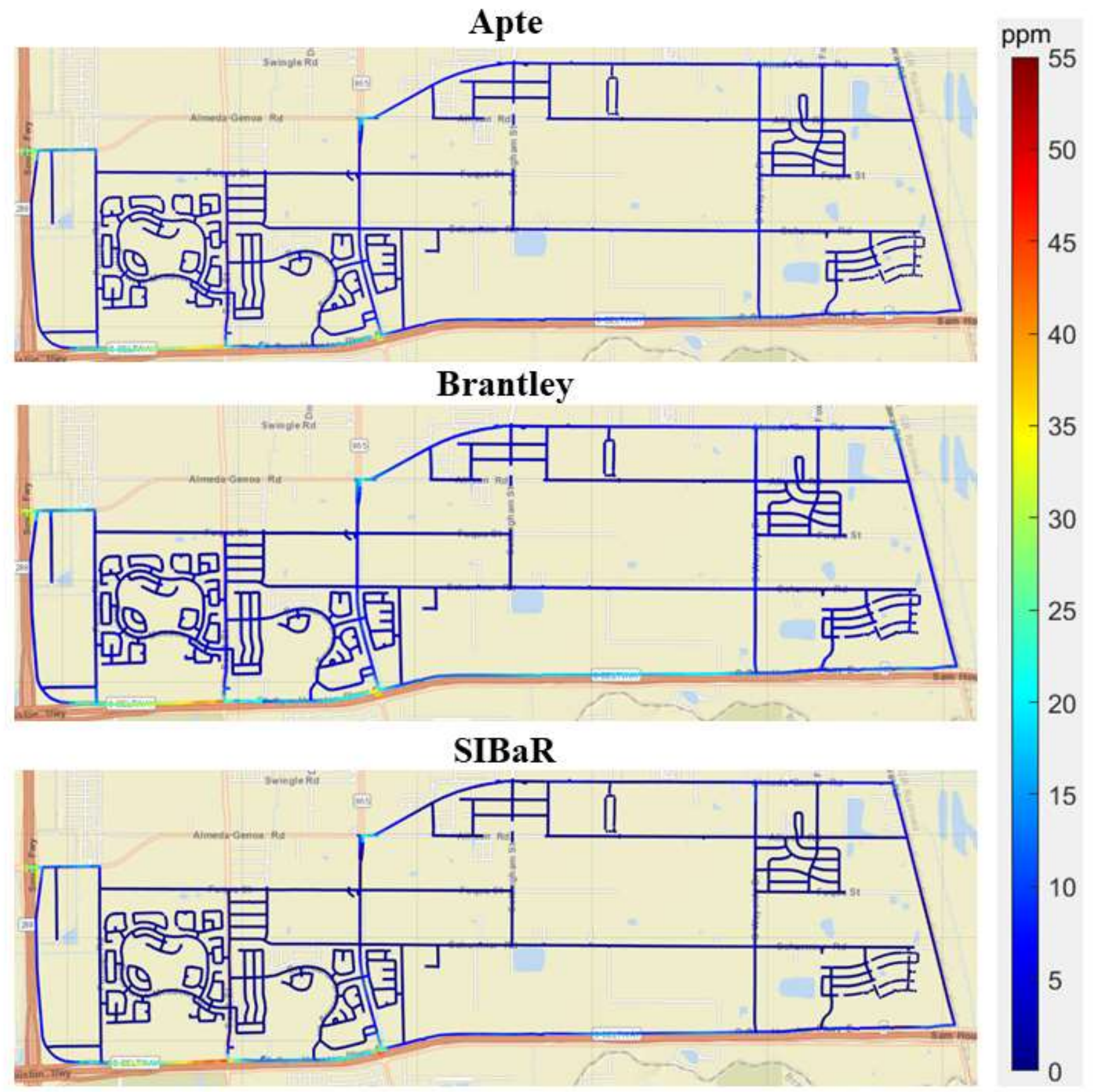

Figure S11. $\mathrm{CO}_{2}$ mapped median source contributions. South Beltway Central. Basemap generated by Matlab geobasemap 'streets' and is hosted by ESRI (Sources: Esri, DeLorme, HERE, USGS, Intermap, iPC, NRCAN, Esri Japan, METI, Esri China (Hong Kong), Esri (Thailand), MapmyIndia, Tomtom). 


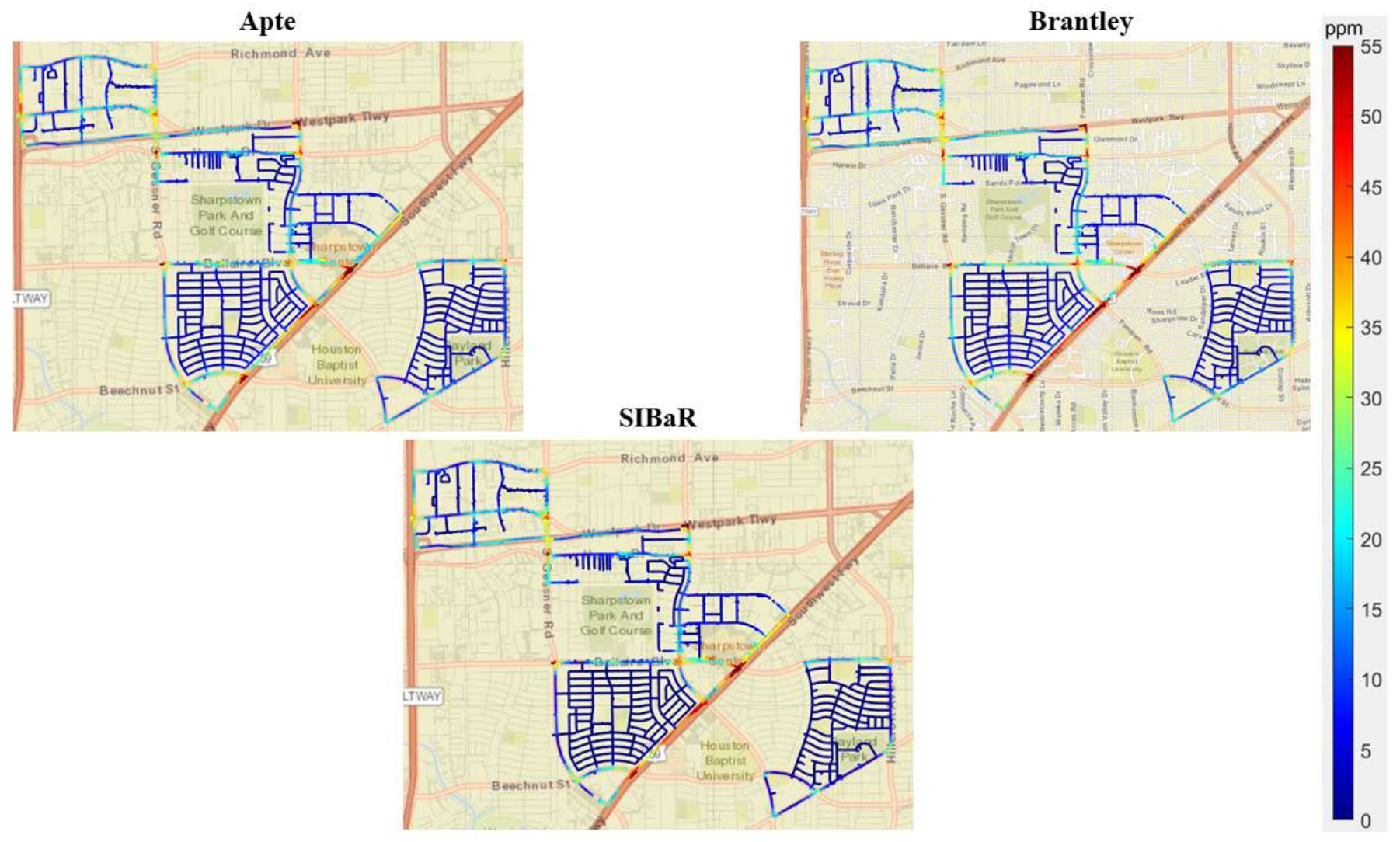

Figure $\mathrm{S12}_{2} \mathrm{CO}_{2}$ mapped median source contributions. Southwest quadrant. Basemap generated by Matlab geobasemap 'streets' and is hosted by ESRI (Sources: Esri, DeLorme, HERE, USGS, Intermap, iPC, NRCAN, Esri Japan, METI, Esri China (Hong Kong), Esri (Thailand), MapmyIndia, Tomtom). 


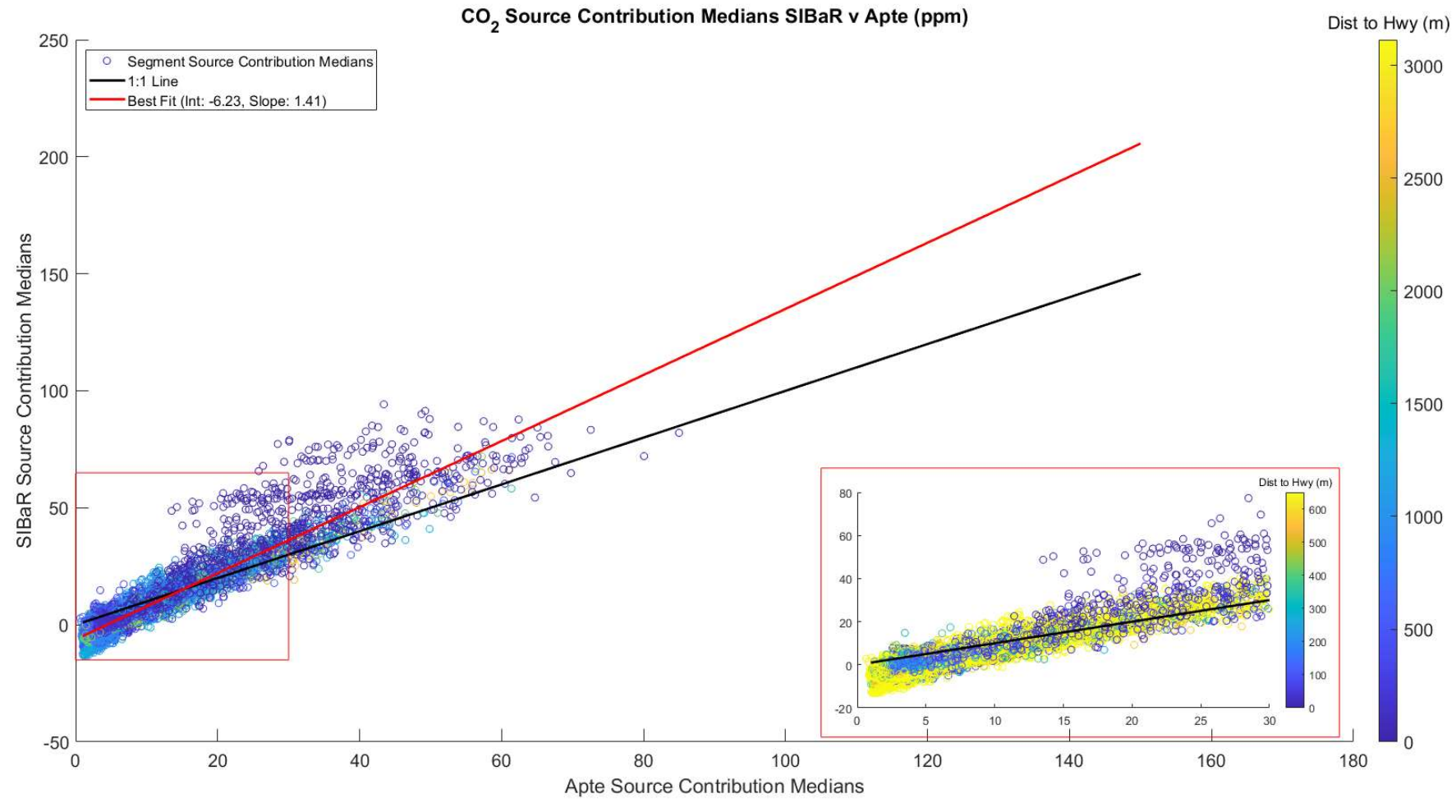

Figure S13. Comparison of $\mathrm{CO}_{2}$ Road Segment Median Source Contributions for SIBaR and Apte.

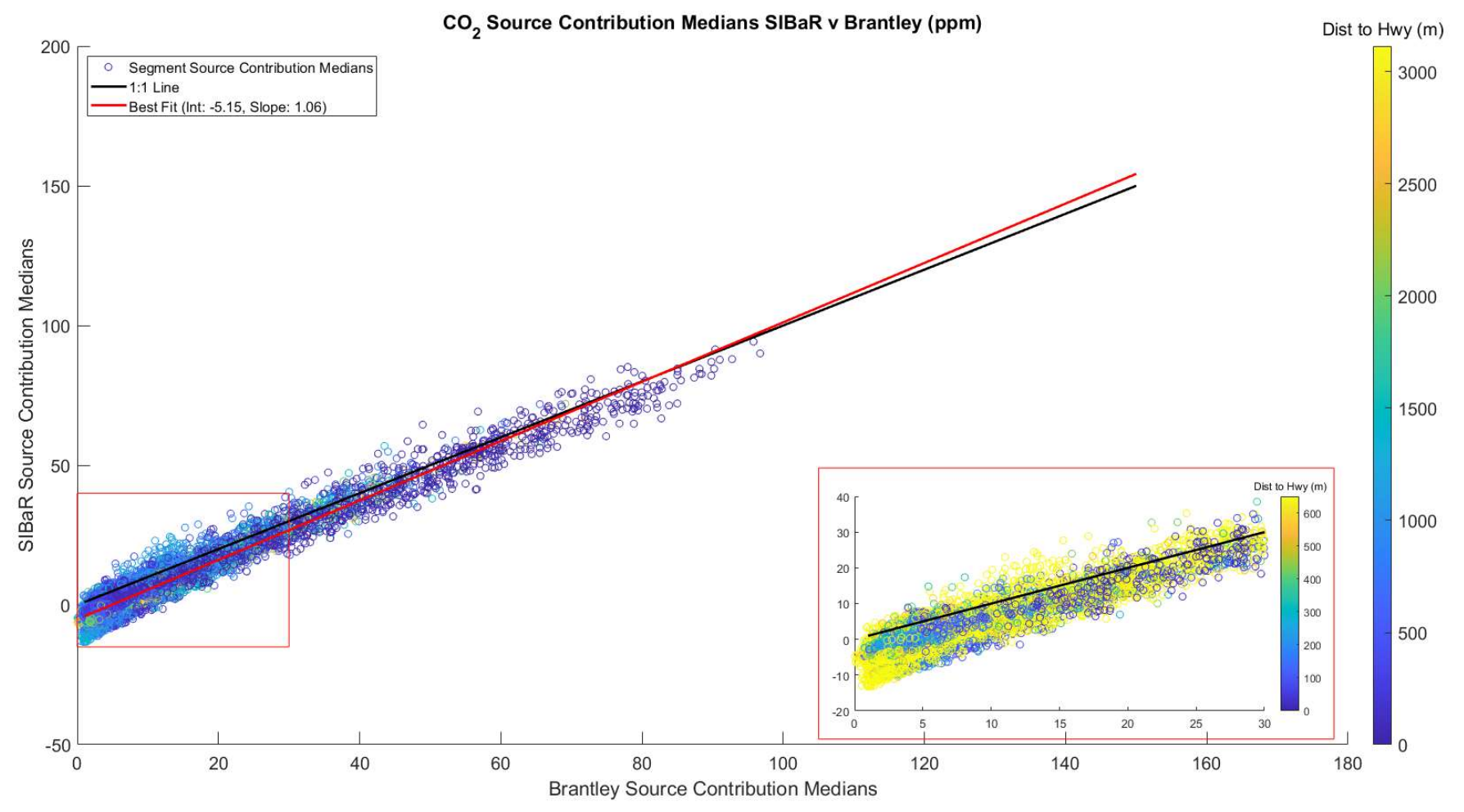

Figure S14. Comparison of $\mathrm{CO}_{2}$ Road Segment Median Source Contributions for SIBaR and Brantley. 


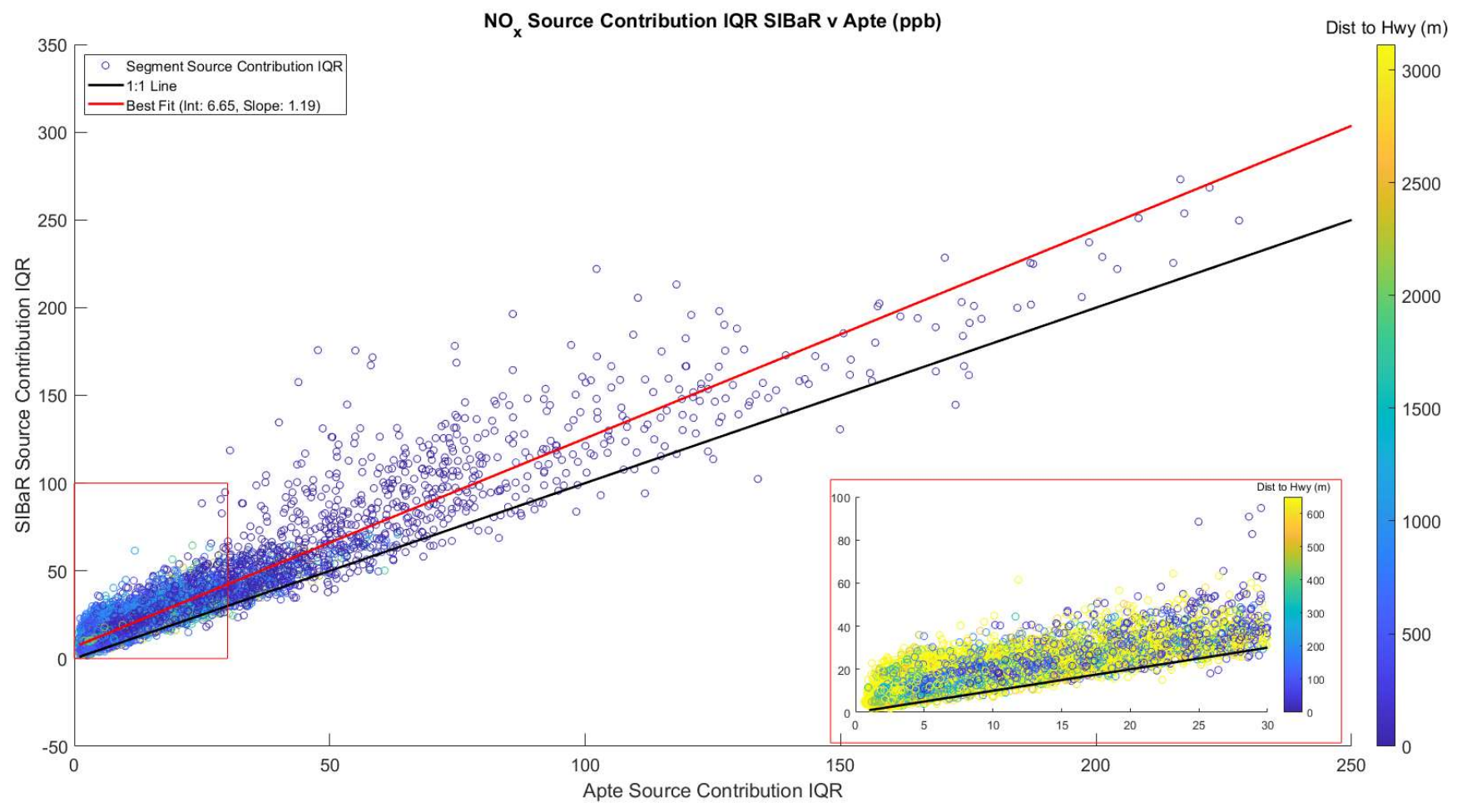

Figure S15. Comparison of NOx Road Segment IQR Source Contribution for SIBaR and Apte.

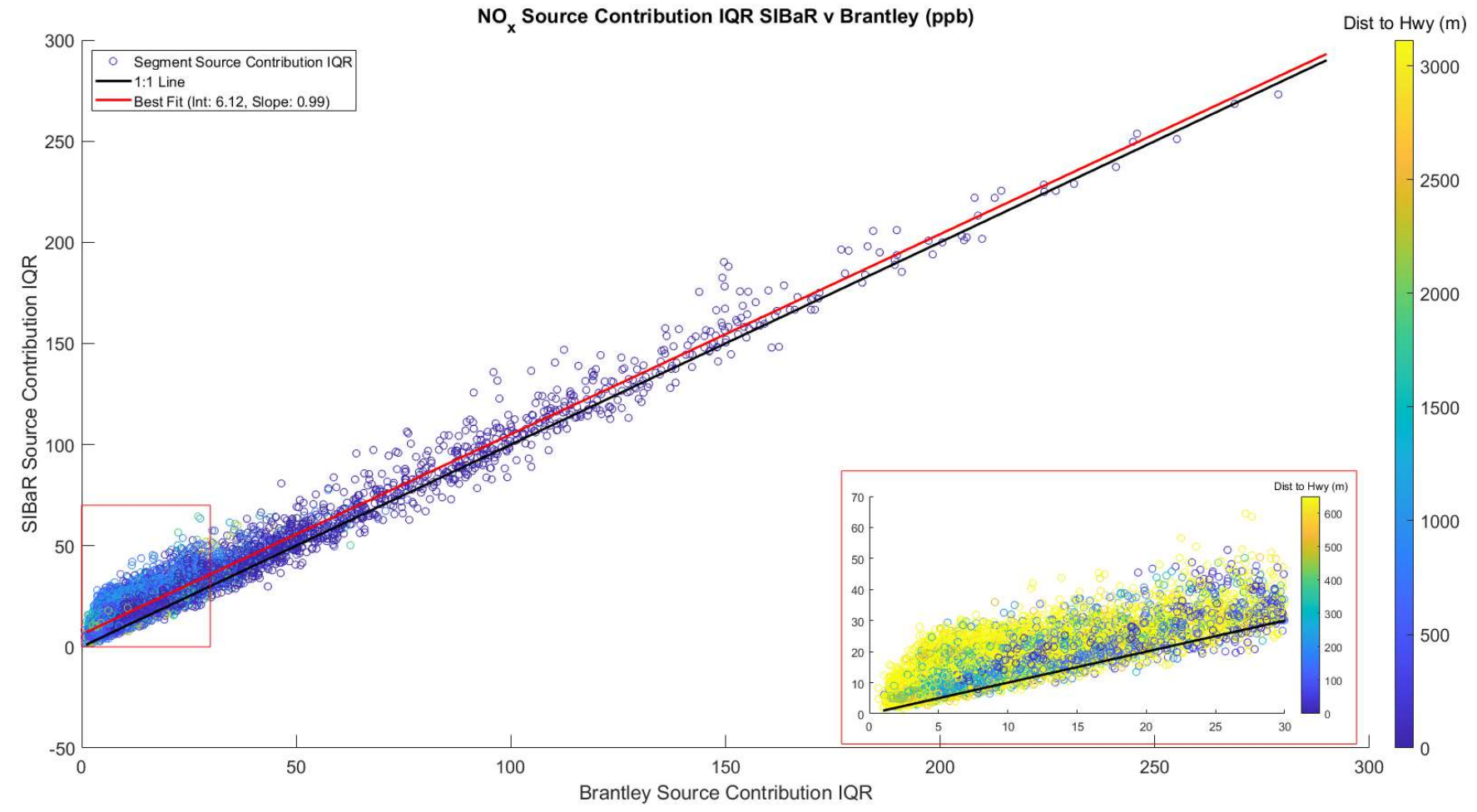

Figure S16. Comparison of NO $\mathrm{x}_{\mathrm{x}}$ Road Segment IQR Source Contribution for SIBaR and Brantley. 


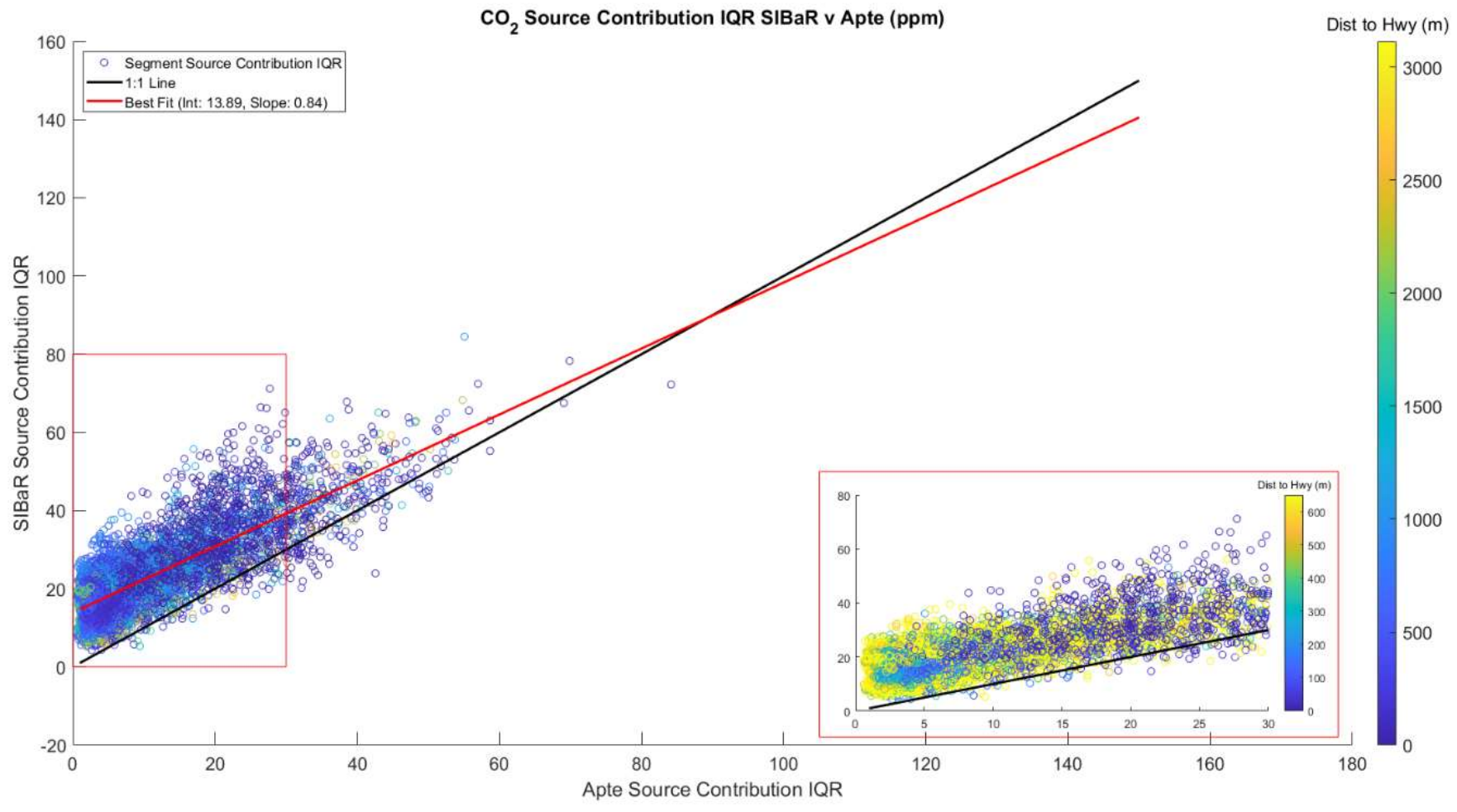

Figure S17. Comparison of $\mathrm{CO}_{2}$ Road Segment IQR Source Contribution for SIBaR and Apte.

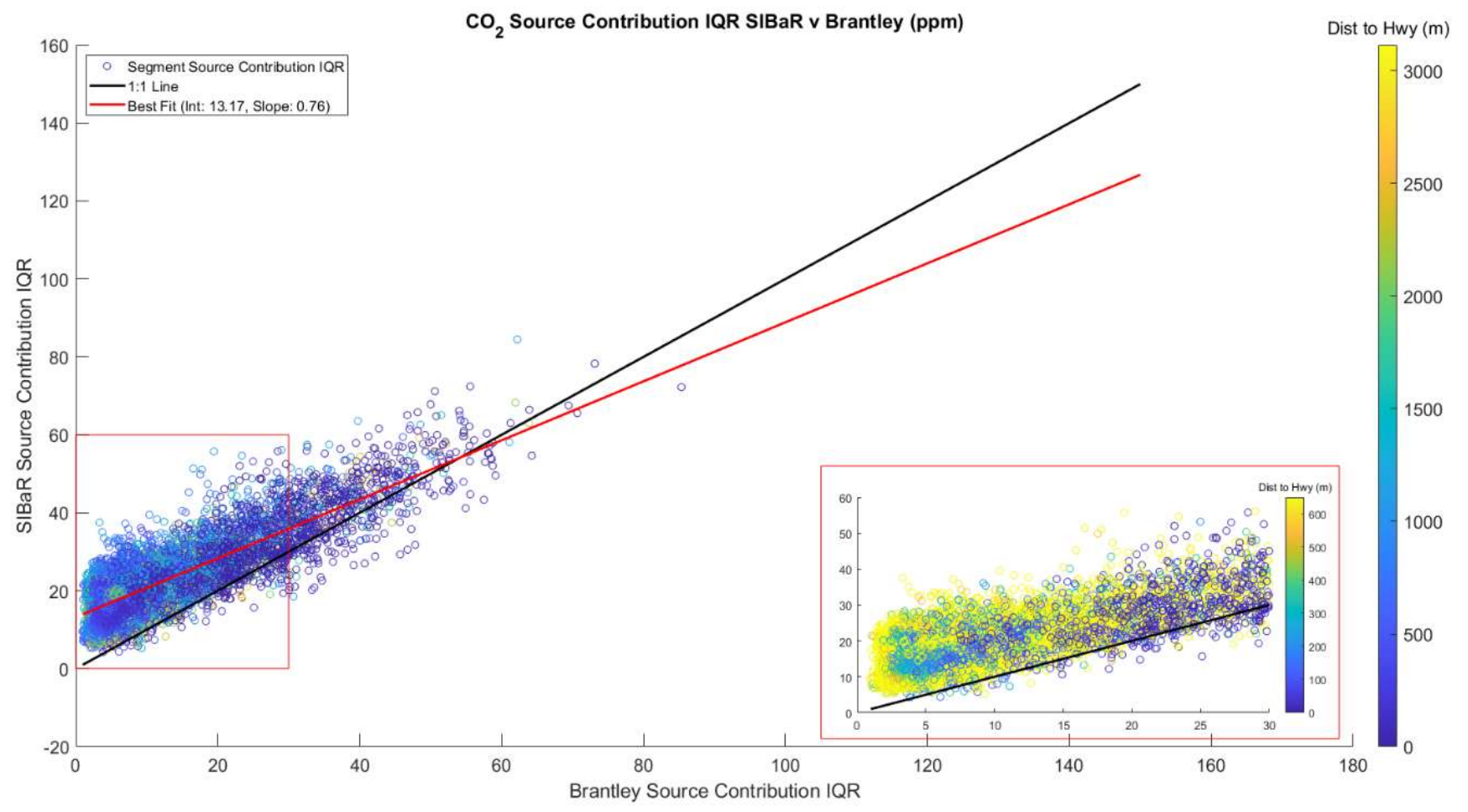

Figure S18. Comparison of $\mathrm{CO}_{2}$ Road Segment IQR Source Contribution for SIBaR and Brantley. 


\section{References}

Census 2010 Tracts, [online] Available from: https://cohgis-mycity.opendata.arcgis.com/datasets/census2010-tracts (Accessed 23 November 2020)

Finding pollution — and who it impacts most — in Houston, Environmental Defense Fund [online] Available from: https://www.edf.org/maps/airqualitymaps/houston/pollution-map/ (Accessed 23 November 2020). 